\title{
Diagnóstico por imagem do tromboembolismo pulmonar agudo*
}

\author{
Imaging of acute pulmonary thromboembolism \\ C. ISABELA S. SILVA, NESTOR L. MÜLLER
}

O diagnóstico do tromboembolismo pulmonar agudo é baseado na probabilidade clínica, uso do dímero $\mathrm{D}$ (quando disponível) e na avaliação por imagem. Os principais métodos de imagem utilizados no diagnóstico são representados por cintilografia ventilação-perfusão, angiografia pulmonar e tomografia computadorizada (TC). $\mathrm{Na}$ última década vários estudos têm demonstrado que a TC espiral apresenta elevada sensibilidade e especificidade no diagnóstico de tromboembolismo pulmonar agudo. Uma melhor avaliação das artérias pulmonares tornou-se possível com a recente introdução dos equipamentos de TC espirais com multidetectores. Vários pesquisadores têm sugerido que a angiografia pulmonar por TC espiral deve substituir a cintilografia na avaliação de pacientes com suspeita clinica de tromboembolismo pulmonar agudo. Os autores discutem os principais métodos de imagem utilizados no diagnóstico de tromboembolismo pulmonar agudo enfatizando o papel da TC espiral.

J Bras Pneumol 2004; 30(5) 474-9
The diagnosis of acute pulmonary thromboembolism is based on the clinical probability, use of D-dimer (when available) and imaging. The main imaging modalities used in the diagnosis are ventilationperfusion (V/Q), scintigraphy, angiography, and computed tomography (CT). In the last decade several studies have demonstrated that spiral CT has a high sensitivity and specificity in the diagnosis of acute pulmonary thromboembolism. The evaluation of the pulmonary arteries has further improved with the recent introduction of multidetector spiral CT scanners. Various investigators have suggested that spiral CT pulmonary angiography should replace scintigraphy in the assessment of patients whose symptoms are suggestive of acute PE. This article discusses the role of the various imaging modalities in the diagnosis of acute pulmonary thromboembolism with emphasis on the role of spiral $\mathrm{CT}$.
Descritores: Diagnóstico por imagem. Embolia pulmonar/ diagnóstico. Angiografia. Pneumopatias/cintilografia. Tomografia computadorizada de emissão de fóton único/ métodos.
Key words: Diagnostic imaging. Pulmonary embolism./ diagnosis. Angiography. Lung diseases/radionuclide imaging. Tomography, emission-computed single-photon/methods. 


\section{INTRODUÇÃO}

Tromboembolismo pulmonar (TEP) é uma entidade clinica comum que resulta em morbidade e mortalidade em um grande número de pacientes. Diagnóstico imediato e correto é importante por causa dos riscos de complicações do TEP e do tratamento com anticoagulantes. A base do diagnóstico é a probabilidade clínica para tromboembolismo pulmonar e 0 uso do dímero $D$ (quando disponível). No entanto, o diagnóstico específico requer avaliação por métodos específicos de imagem ${ }^{(1)}$.

Por muitos anos, a cintilografia ventilaçãoperfusão (V/Q) representou o principal método de imagem utilizado na avaliação de pacientes com suspeita clínica de TEP(2). A cintilografia de alta probabilidade fornece suficiente confiabilidade para confirmar o diagnóstico de TEP, enquanto que exame normal ou perto do normal, exclui, seguramente, o diagnóstico. Infelizmente só um terço dos pacientes pertencem a uma destas categorias; nos restantes dois terços dos pacientes os resultados da cintilografia são inconclusivos ${ }^{(2)}$. Deve-se ressaltar também que, no Brasil, a cintilografia pulmonar não está disponível diariamente, limitando ainda mais o seu uso.

Tradicionalmente a angiografia pulmonar foi considerada o método padrão ouro para diagnóstico de TEP(2,3). Todavia, a angiografia pulmonar é um método invasivo, disponível em poucos centros e utilizado cada vez menos na avaliação destes pacientes, mesmo quando os outros exames não são conclusivos ${ }^{(4,5)}$.

A introdução da tomografia computadorizada (TC) espiral no início dos anos 90 tornou possível avaliar todo o tórax num curto espaço de tempo, bem como analisar as artérias pulmonares durante 0 pico máximo de opacificação pelo meio de contraste. Vários estudos demonstraram elevada sensibilidade e especificidade da TC espiral para o diagnóstico de TEP ${ }^{(6-8)}$. A acurácia da TC aumentou ainda mais com 0 recente advento dos equipamentos de TC espiral com multidetectores que permitem uma melhor avaliação das artérias pulmonares segmentares e subsegmentares. Em diversos centros, a TC espiral tornou-se a modalidade de escolha para o diagnóstico de TEP.

0 objetivo deste trabalho é discutir as indicações e limitações dos principais métodos de imagem utilizados no diagnóstico de tromboembolismo
Siglas e abreviaturas utilizadas neste trabalho:

TC - Tomografia Computadorizada

TEP - Tromboembolismo Pulmonar Agudo

V/Q - Ventilação-Perfusão

US - UItrassom

TVP - Trombose Venosa Profunda

pulmonar agudo com ênfase na tomografia computadorizada espiral.

\section{Cintilografia pulmonar:}

O diagnóstico do TEP na cintilografia é baseado na presença de ventilação em regiões sem perfusão distais a êmbolos obstrutivos. Os achados cintilográficos são classificados em termos de probabilidade da presença de embolismo em cintilograma, de alta probabilidade, probabilidade intermediária, baixa probabilidade, quase normal ou muito baixa probabilidade, e normal. Os exames de cintilografia de alta probabilidade fornecem suficiente confiabilidade para confirmar 0 diagnóstico de TEP enquanto que os exames de muito baixa probabilidade e normais permitem excluir o diagnóstico. A grande limitação da cintilografia $\mathrm{V} / \mathrm{Q}$ está relacionada ao elevado número de pacientes em que os achados não são conclusivos. 0 estudo PIOPED (Prospective Investigation of Pulmonary Embolism Diagnosis), maior estudo prospectivo de avaliação de pacientes com suspeita clinica de TEP, demonstrou que em dois terços dos pacientes que realizaram cintilografia $V / Q$, ela foi incapaz de estabelecer ou excluir TEP. Neste estudo, nos exames indeterminados, presentes em 39\% (364 de 931) dos pacientes, encontrou-se uma incidência de $30 \%$ de TEP, e nos exames de baixa probabilidade, identificados em $34 \%$ dos pacientes (312 de 931), observou-se uma incidência de $14 \%^{(2)}$. Baseados nesses achados, os autores concluíram que exames indeterminados e de baixa probabilidade (ou seja, dois terços dos exames de V/Q do estudo PIOPED), foram incapazes de estabelecer ou excluir TEP. Uma outra limitação da cintilografia é a discordância entre diferentes observadores na interpretação dos exames. No estudo PIOPED embora tenha sido encontrado boa concordância entre observadores com grande experiência para os exames V/Q normais e de alta probabilidade, notou-se entre os observadores, 25 -30\% de discordância na interpretação de exames de baixa probabilidade. (2) 


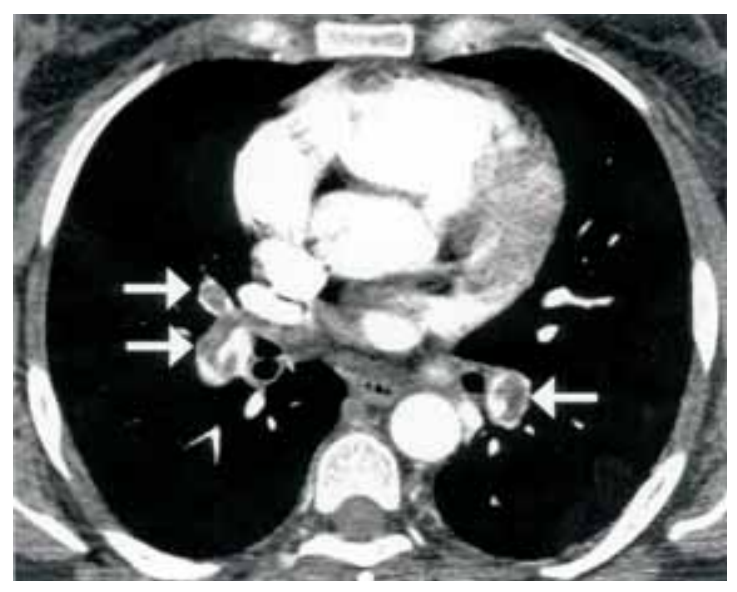

Figura 1- TEP agudo em paciente do sexo feminino, 46 anos. TC espiral em multidetector revela falhas de enchimento parcial nas artérias do lobo médio e lobos inferiores (setas).

\section{Angiografia pulmonar:}

$\mathrm{Na}$ angiografia pulmonar introduz-se um cateter por via endovenosa na artéria pulmonar proximal e 0 meio de contraste é injetado rapidamente. A técnica oferece uma elevada resolução espacial, permitindo avaliar diretamente o sistema arterial pulmonar. Os achados de defeito de enchimento da coluna de contraste são típicos de TEP. Todavia, a angiografia pulmonar é um método invasivo, com $5 \%$ de riscos de complicações cardíacas e pulmonares, e mortalidade de $0,3 \%{ }^{[3]}$. Devido aos riscos potencias, existe considerável relutância por parte dos clínicos e radiologistas na realização de angiografia pulmonar para 0 diagnóstico de TEP. Mesmo nos grandes centros acadêmicos dos Estados

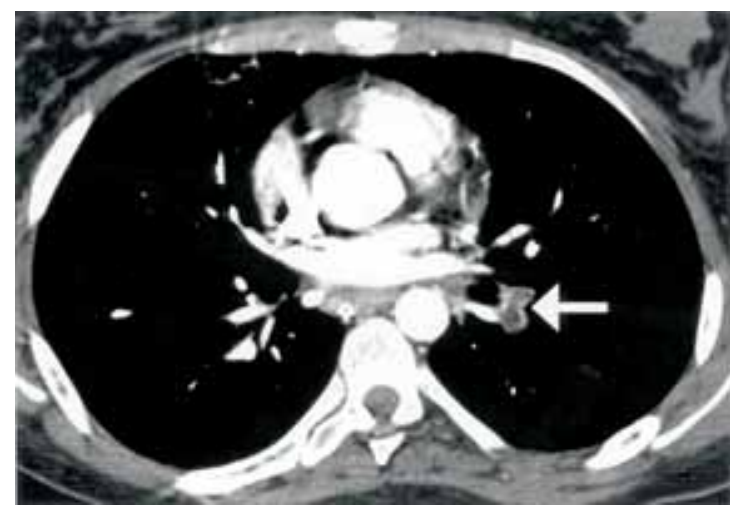

Unidos e da Inglaterra, com disponibilidade de equipamento adequado e pessoal especializado, estima-se que somente 5 a $15 \%$ dos pacientes com exames de cintilografia indeterminados, são submetidos à angiografia pulmonar ${ }^{(4,5)}$.

\section{Tomografia computadorizada espiral}

Interpretação das Imagens:

Os achados característicos de TEP agudo são:

1) defeito de enchimento parcial central ou periférico circundado por um pequeno halo de contraste (Figura 1);

2) completo defeito de enchimento com obliteração de todo 0 vaso avaliado ${ }^{(6-8)}$.

Num evento de TEP agudo, as artérias pulmonares quando completamente obstruídas geralmente têm diâmetro aumentado (Figura 2A, 2B). Nos exames de pesquisa de TEP deve-se avaliar os achados vasculares e parenquimatosos. A avaliação da janela de pulmão é importante não apenas para identificar as artérias pulmonares devido sua proximidade com o brônquio, mas também para avaliar sinais complementares que podem ser úteis em sugerir o diagnóstico de TEP(9,10). 0 achado complementar de maior utilidade é a identificação de opacidade de feitio triangular e base pleural (Figura 3). Esta opacidade reflete a presença de hemorragia ou infarto distal ao embolismo. Atelectasias lineares também são identificadas com freqüência elevada nos exames de TC de pacientes com TEP agudo. Outros sinais tomográficos tais como áreas de atenuação reduzida e efusão pleural são de pouca utilidade em distinguir pacientes com e sem TEP agudo ${ }^{(9,10)}$.
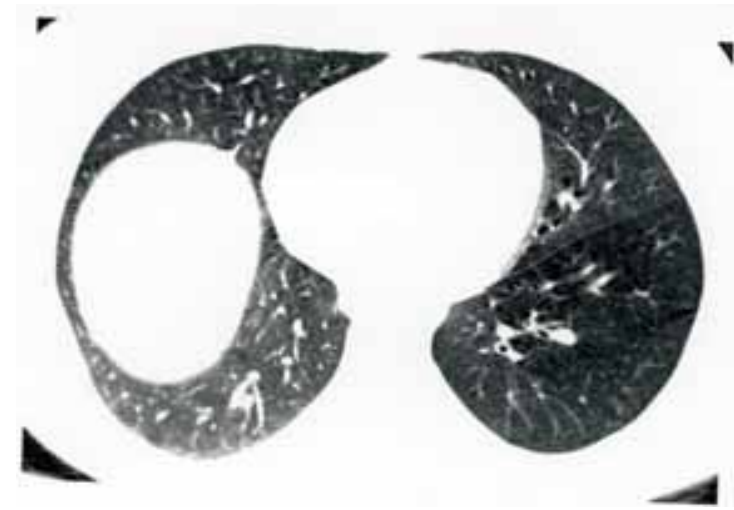

Figura 2A- TEP agudo em paciente do sexo feminino, 15 anos, vítima de acidente automobilístico. TC espiral em multidetector mostra defeitos de enchimento completo em artérias do lobo inferior esquerdo, que também apresentam calibre aumentado. B Janela de pulmão revela diminuição da atenuação e vascularização pulmonar no lobo inferior esquerdo (sinal de Westermark) secundário a presença de trombo oclusivo nas artérias correspondentes. 


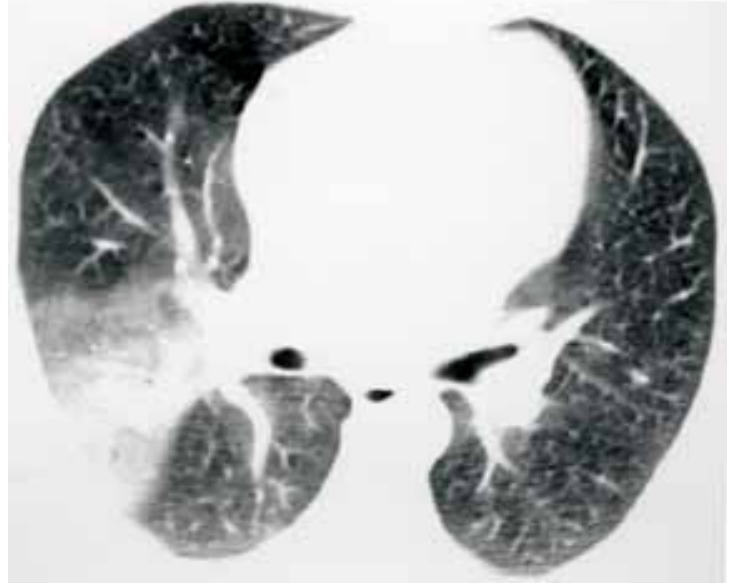

Figura 3- TEP agudo em paciente do sexo masculino, 37 anos. Imagem de TC espiral revela opacidade pulmonar de feitio triangular e base pleural no segmento posterior do lobo superior direito, compatível com hemorragia pulmonar.

Possíveis problemas relacionados à técnica, anatomia e ao paciente podem levar à falsa interpretação nas imagens de TC. Problemas técnicos ocorrem em 1 a $5 \%$ dos exames, e na grande maioria são decorrentes de artefatos de movimento em pacientes dispnéicos ou de opacificação inadequada dos vasos pelo meio de contraste.

Os tecidos linfáticos e conectivos localizados adjacentes às artérias pulmonares podem mimetizar a aparência de TEP. Este problema pode ser minimizado através de revisão cautelosa das imagens e uso de recursos adicionais de imagens, tais como o modo cine-view (usado por nós rotineiramente) e reconstruções multiplanares.

\section{Acurácia Diagnóstica da TC Espiral}

Os relatos da acurácia diagnóstica da TC espiral têm variado de acordo com a técnica utilizada e da população de pacientes avaliados. De modo geral, esses estudos têm mostrado uma sensibilidade de $90 \%$, especificidade de $90 \%$, valor preditivo positivo de $93 \%$ e valor preditivo negativo de $94 \%$ para TC espiral na avaliação de tromboembolismo pulmonar agudo(11).

Deve-se salientar que embora a TC espiral tenha uma elevada sensibilidade para detectar trombos nas artérias pulmonares centrais (Figura 4), lobares e segmentares, ela tem valor limitado no diagnóstico de trombo subsegmentar. Todavia, o significado clínico de trombo subsegmentar

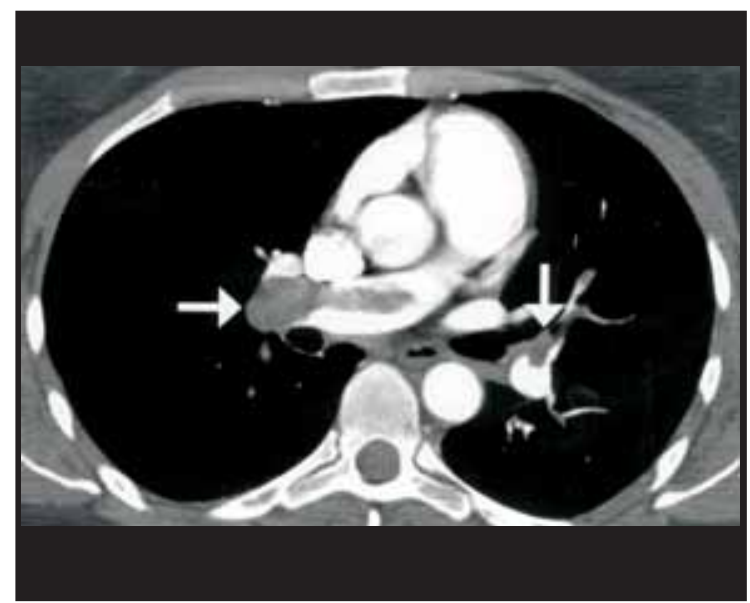

Figura 4- TEP agudo em paciente do sexo feminino, 49 anos. Imagem de TC em multidetector evidencia falhas de enchimento na artéria pulmonar direita, artéria interlobar direita e artéria interlobar esquerda com extensão para a artéria lingular (setas).

isolado é controverso. Além de que, embora a angiografia pulmonar seja considerada o padrão ouro para diagnóstico de TEP, a concordância na avaliação das artérias pulmonares subsegmentares, mesmo entre observadores com grande experiência com a técnica é só de $66 \%{ }^{(3)}$. Resultados preliminares em modelos experimentais têm mostrado que a TC espiral é comparável a angiografia pulmonar no diagnóstico de TEP subsegmentar ${ }^{(12)}$. No entanto, recentes trabalhos indicam que a acurácia da TC espiral no diagnóstico de trombo subsegmentar tem aumentado com 0 uso de cortes mais finos, tais como colimação de 1 ou $2 \mathrm{~mm}$ e uso de equipamentos de TC com multidetectores $^{(13-15)}$. Os equipamentos de TC espiral com multidetectores permitem avaliar todo o tórax em poucos segundos com cortes de $1 \mathrm{~mm}$ de espessura e dessa maneira uma melhor avaliação das artérias segmentares e subsegmentares (Figura 5). Outra vantagem é a realização de imagens multiplanares de melhor qualidade que facilitam a confirmação ou exclusão do diagnóstico de TEP, nos casos em que o diagnóstico foi inconclusivo nas imagens convencionais (Figura 6) ${ }^{(13)}$

Vários trabalhos demonstraram que uma TC espiral negativa, desde que as imagens sejam de boa qualidade, é suficiente para excluir TEP, e que 0 prognóstico dos pacientes com TC espiral negativa e não tratados com anticoagulante, é semelhante aos descritos para exames de angiografia e cintilografia 

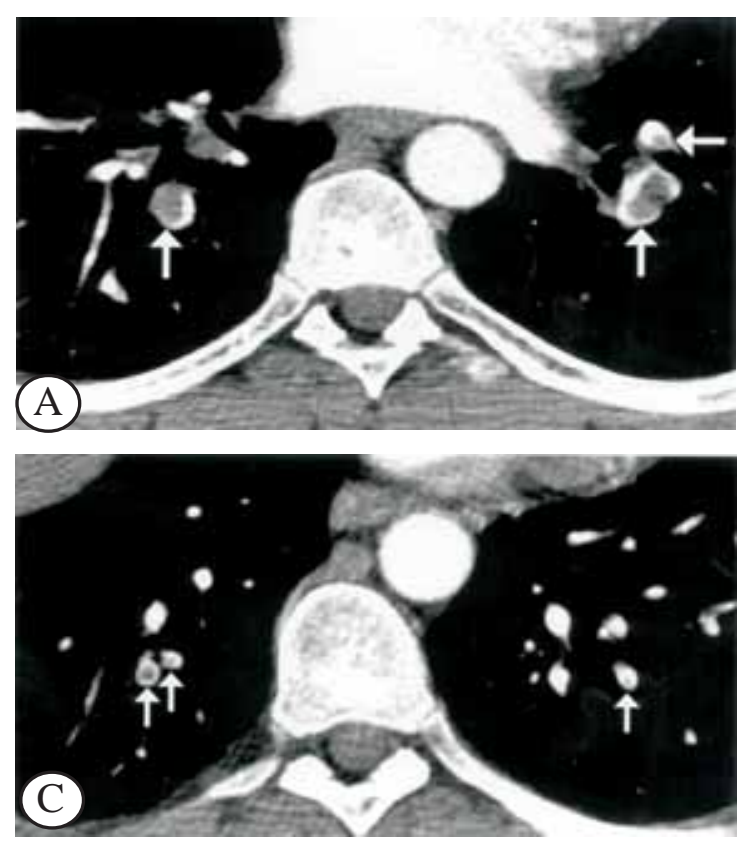

negativos ${ }^{(15,16)}$. Goodman et al compararam os achados de 198 pacientes com TC espiral negativas com os achados de cintilografia negativas (normal ou de baixa probabilidade) em 350 pacientes ${ }^{(15)}$. Após três meses de follow-up, TEP subsegmentar foi encontrado em $1 \%$ dos pacientes do grupo de TC espiral comparado a 1,5\% dos pacientes do grupo

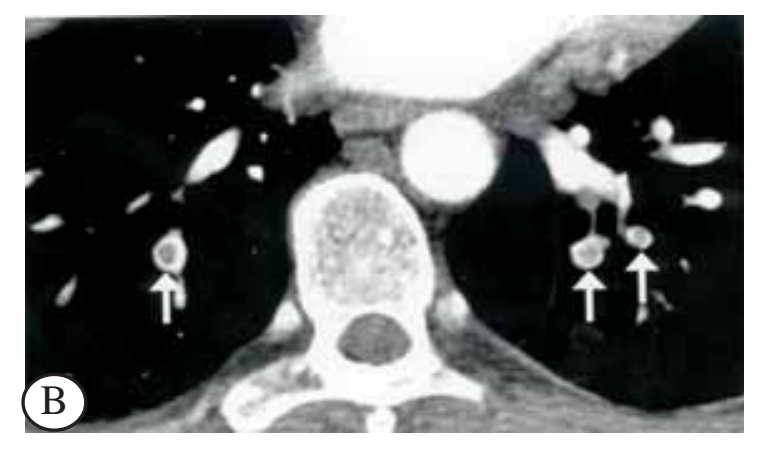

Figura 5- TEP agudo em artérias segmentares e subsegmentares. A. TEP agudo em artérias segmentares e subsegmentares. Imagem de TC em multidetector ao nível dos lobos inferiores demonstra defeitos de enchimento parciais ao nível das artérias segmentares proximais (setas).B. Imagem de TC em nivel mais caudal demonstra defeitos parciais de enchimento ao nível das artérias segmentares distais (setas). C. Imagem de TC mais inferior que a anterior mostra falhas de enchimento parciais ao nível das artérias subsegmentares (setas).

de cintilografia normal ou de baixa probabilidade (estatisticamente insignificante). Swensen revisou 1512 pacientes consecutivos encaminhados para TC com suspeita clinica de TEP agudo ${ }^{(16)}$. Novecentos e noventa e três desses pacientes tiveram exames de TC interpretados como negativos para TEP agudo e não receberam anticoagulante. Somente $0,5 \%$ desses
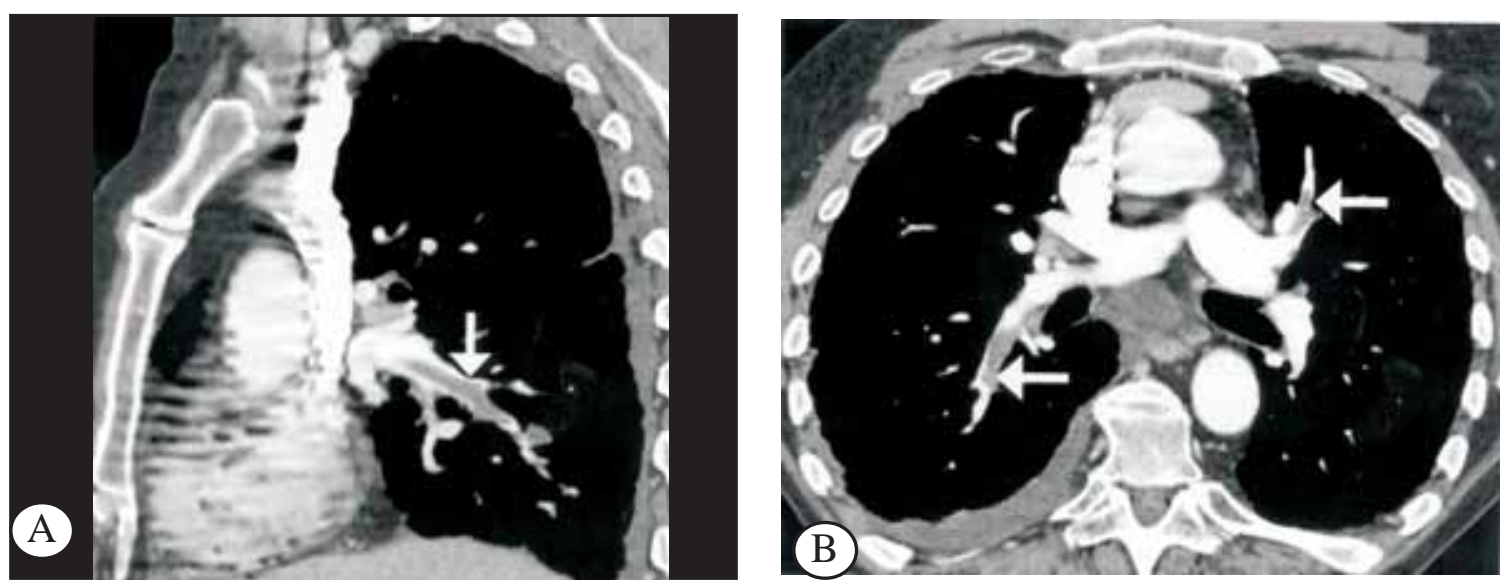

Figura 6A- TC espiral em multidetector com reconstruções multiplanares no TEP agudo.A reformatação sagital demonstra falha de enchimento parcial na artéria pulmonar interlobar direita com extensão para a artéria pulmonar do lobo inferior. B. Reconstrução axial curva evidencia defeitos de enchimentos parciais no segmento arterial superior direito e na artéria lingular (setas). Também são identificados, pequena efusão pleural à direita e linfonodomegalia subcarinal. 
pacientes desenvolveram tromboembolismo venoso num follow-up de 3 meses. Estes resultados são semelhantes aos descritos em pacientes com suspeita de TEP que tiveram angiografia negativa. Os autores concluíram que é seguro não utilizar terapia anticoagulante em pacientes com TC espiral negativa e sem evidência clínica de trombose venosa profunda ${ }^{(16)}$

\section{Algoritmo diagnóstico}

A literatura mais recente recomenda o seguinte algoritmo diagnóstico para a avaliação por imagem de pacientes com suspeita clínica de TEP agudo ${ }^{(17)}$ :

1.Todos os pacientes devem ter uma radiografia do tórax, cujo principal papel é excluir anormalidades tais como pneumonia que podem mimetizar TEP clinicamente.

2.Pacientes com sintomas ou sinais de trombose venosa profunda devem ser submetidos à avaliação dos membros inferiores, sendo o ultrassom (US) com Doppler colorido geralmente o método recomendado. Caso o exame de US com Doppler seja positivo para TVP, o paciente pode ser considerado como tendo TEP e geralmente não requer outra investigação.

3.Pacientes com suspeita clinica de TEP e sem sinais ou sintomas de TVP devem ser submetidos à angiografia pulmonar por TC espiral. Salienta-se que a realização desse exame requer a utilização de contraste iodado. Pacientes que têm contraindicação à utilização do meio de contraste devem realizar cintilografia pulmonar. Deve-se também salientar que a cintilografia continua sendo 0 método de imagem principal em centros que não possuem TC espiral.

4.Pacientes com exames de TC de má qualidade e negativos para TEP, mas com elevada suspeita clínica, devem ser submetidos à angiografia pulmonar.

\section{REFERÊNCIAS:}

1. Wells PS, Rodger M. Diagnosis of pulmonary embolism: when is imaging needed? Clin Chest Med. 2003;24:13-28.

2. The PIOPED Investigators. Value of ventilation-perfusion scan in acute pulmonary embolism. Results of the prospective investigation of pulmonary embolism diagnosis (PIOPED). J AMA 1990; 263: 2753-9.
3. Stein PD, Athanasoulis C, Alavi A. Complications and validity of pulmonary angiography in acute pulmonary embolism. Circulation 1992; 85: 462-8.

4. Schluger $\mathrm{N}$, Henschke $\mathrm{Cl}$, King $\mathrm{T}$. Diagnosis of pulmonary embolism at a large teaching hosptital. J. Thorac Imag 1994; 9: 180-4

5. Cooper TJ, Hayward MWJ, Hartog M. Survery on the use of pulmonary scintigraphy and angiography for supspected pulmonary thromboembolism in the UK. Clin Radiol 1991; 43: 243-5

6. Remy-Jardin M, Remy J, Deschildre F. Diagnosis of pulmonary embolism with spiral CT: Comparison with pulmonary angiography and scintigraphy. Radiology 1996; 200: 699-706.

7. Mayo JR, Remy-Jardin M, Müller NL. Pulmonary embolism: prospective comparison of spiral CT with ventilation-perfusion scintigraphy. Radiology 1997; 205: 447-52.

8. Qanadli SD, El Hajjam M, Mesurolle B. Pulmonary embolism detection: prospective evaluation of dualsection helical CT versus selective pulmonary arteriography in 157 patients. Radiology 2000; 217:447-55.

9. Coche EE, Müller NL, Kim W, Wiggs BR, Mayo JR. Acute pulmonary embolism: ancillary findings at spiral CT. Radiology 1998;207:753-8.

10. Shah AA, Davis SD, Gamsu G, Intriere L. Parenchymal and pleural findings in patients witth and patients without acute pulmonary embolism detected at spiral CT. Radiology 1999:211:147-53.

11. Maki DD, Gefter WB, Alavi A. Recent advances in pulmonary imaging. Chest 1999;116:1388-402.

12. Baile EM, Mayo JR, King GG, Müller NL, Coche EC, Paré PD. Contrast-enhanced spiral CT is comparable to pulmonary angiography for the diagnosis of pulmonary embolism. Am J Resp Crit Care Med 2000; 161:1010- 5 .

13. Remy-J ardin M, Mastora I, Remy J. Pulmonary embolus imaging with multislice CT. Radiol Clin North America 2003; 41:507-19

14. Patel S, Kazerooni EA, Cascade PN. Pulmonary embolism: optimization of small pulmonary artery visualization at multi-detector row CT. Radiology 2003; 227:455-60.

15. Goodman LR, Lipchik RJ, Kuzo RS, Liu Y, McAuliffe TL, O'Brien DJ. Subsequent pulmonary embolism: risk after a negative helical CT pulmonary angiogram prospective comparison with scintigraphy. Radiology 2000; 215:535-42.

16. Swensen SI, Sheedy PF, Ryu JH, Picket al. Outcomes after withholding anticoagulation from patients with suspected acute pulmonary embolism and negative computed tomographic findings: a cohort study. Mayo Clinic Proceedings 2002; 77:130-8.

17. Powell T, Müller NL. Imaging of Acute Pulmonary Thromboembolism: Should Spiral Computed Tomography Replace the V/Q Scan? Clin Chest Med. 2003; 24:29-38 